\title{
Teilzeitbeschäftigung - Ausmaß und Bestimmungsgründe der Erwerbsübergänge von Frauen
}

\author{
Claudia Vogel
}

Angenommen: 8. März 2009 / Online veröffentlicht: 9. Mai 2009

(C) Institut für Arbeitsmarkt- und Berufsforschung 2009

Zusammenfassung Bislang überwiegt das Normalarbeitsverhältnis in Form der kontinuierlichen Vollzeitbeschäftigung auf dem deutschen Arbeitsmarkt. Allerdings ist spätestens seit der Wiedervereinigung eine stetige Zunahme der Teilzeitbeschäftigung zu beobachten. Der vorliegende Beitrag untersucht auf der Grundlage des Sozio-ökonomischen Panels (SOEP) der Jahre 1991 bis 2006, in welchem Umfang Teilzeitarbeit von Frauen und Männern ausgeübt wird, wie die Beteiligung nach Altersgruppen variiert und mittels logistischer Regressionsmodelle - welche Merkmale die Aufnahme einer Teilzeit- bzw. Vollzeitbeschäftigung bei Frauen begünstigen. Obwohl auch die Teilzeitquoten der männlichen Beschäftigten gestiegen sind - fast jeder zehnte Arbeitnehmer und knapp die Hälfte der Arbeitnehmerinnen sind teilzeitbeschäftigt - stellen Frauen die überwiegende Mehrheit der Teilzeitbeschäftigten: vier von fünf Teilzeitbeschäftigten sind weiblich. Überdurchschnittlich häufig arbeiten Frauen über 35 Jahren in Teilzeit. Außerdem sind ältere Arbeitnehmer sowie Berufseinsteiger besonders von der Zunahme der Teilzeit betroffen. Bei Frauen sprechen sowohl Mutterschaft als auch Ehe für die Aufnahme einer Teilzeitstelle. Allerdings nehmen ostdeutsche Frauen seltener eine Teilzeitstelle an als westdeutsche, und zwar auch dann, wenn sie Kinder haben. Darüber hinaus ist Teilzeitbeschäftigung für ostdeutsche Arbeitnehmerinnen häufiger ein Sprungbrett in eine Vollzeitanstellung. Offensichtlich kann Teilzeiterwerbstätigkeit der besseren Vereinbarkeit von Familie und Beruf dienen, allerdings wird dieses Arrangement eher von Frauen und Müttern in Westdeutschland gelebt.

C. Vogel (『)

Hochschule Vechta, Institut für Gerontologie,

Zentrum Altern und Gesellschaft,

Driverstr. 22, 49377 Vechta, Deutschland

E-Mail: claudia.vogel@uni-vechta.de
Part-time employment - prevalence and determinants of employment transitions among women

Abstract The labour market in Germany is predominated by the full-time standard employment relationship. Nevertheless, since the reunification at the latest we observe a continuous increase in part-time employment. On the basis of the German Socio-Economic Panel (GSOEP) from 1991 to 2006, we investigate how many women and men do work part-time, how participation in part-time employment varies by age groups and - estimating logistic regression models - which factors facilitate the transition to work either part-time or full-time amongst women. The proportion of part-timers also increased amongst male employees almost one in ten male employees is a part-timer and just under half of female employees work part-time - however, women are predominant amongst part-timers: four in five part-timers are female. Above-average shares of part-time employment show amongst women aged 36 and elder. Furthermore, elderly employees as well as labour market entrants have experienced a significant increase in the prevalence of part-time employment. For women, both motherhood and marriage increase the likelihood to work part-time. However, women from East Germany are less likely to work part-time than are those from West Germany, and that is also the case when they have children. In addition, part-time employment permits transitions into full-time positions more often amongst employees from East Germany. Apparently, part-time employment helps to combine family and working life continuously, but this arrangement is more frequently chosen by mothers in West Germany.

\section{Einleitung}

Eine dauerhafte Vollzeitbeschäftigung ermöglicht Arbeitnehmerinnen und Arbeitnehmern soziale und materielle 
Partizipationschancen. Dies gilt sowohl für die Sicherung des aktuellen Lebensstandards als auch im Hinblick auf zukünftige Perspektiven, etwa aufgrund erworbener Ansprüche in der gesetzlichen Rentenversicherung oder durch die Möglichkeiten zur privaten Altersvorsorge. Empirisch überwiegt in der Bundesrepublik bislang die in der Regel unter dem Begriff des Normalarbeitsverhältnisses gefasste kontinuierliche Vollzeitbeschäftigung. Allerdings ist zum Ende des 20. Jahrhunderts, spätestens seit der Wiedervereinigung, eine stetige Zunahme flexibler Beschäftigungsverhältnisse - insbesondere der Teilzeitbeschäftigung $-\mathrm{zu}$ beobachten, die sich im Hinblick auf die Dauer und die Kontinuität vom Normalarbeitsverhältnis abheben (vgl. z. B. Tálos 1999; Keller u. Seifert 2006).

Zwischen 1991 und 2004 stieg die Zahl der Teilzeitbeschäftigten von 4,7 auf 7,2 Millionen (Statistisches Bundesamt 2005). Das entspricht bereits fast einem Viertel aller Beschäftigten (23\%), während dieser Anteil 1991 noch bei $14 \%$ lag. Teilzeitbeschäftigung ist die am weitesten verbreitete Form der flexiblen Beschäftigung und gewinnt vor allem mit dem ebenfalls zu konstatierenden Anstieg der Frauenerwerbstätigkeit an Bedeutung, weil Frauen die große Mehrheit der Teilzeitbeschäftigten stellen. Zudem wird der Trend zur Zunahme aufgrund des Rechts auf Teilzeit, das im Gesetz über Teilzeitarbeit und befristete Arbeitsverträge geregelt ist und seit seiner Einführung 2001 vermehrt wahrgenommen wird, wohl auch zukünftig anhalten (vgl. z. B. Wagner 2004).

Da Teilzeitarbeit sicherlich nicht für alle Arbeitsmarktgruppen in gleichem Maße attraktiv ist, untersucht der vorliegende Beitrag, in welchem Umfang Teilzeit von Frauen und Männern ausgeübt wird, wie die Beteiligung nach Altersgruppen variiert und, ausschließlich für die weiblichen Beschäftigten, welche Merkmale die Wahrscheinlichkeit eines Erwerbsübergangs in Teilzeit bzw. in Vollzeit beeinflussen. Der Fokus der Betrachtung liegt auf den familialen Determinanten der Übergänge, da die Vereinbarkeit von Familie und Beruf als Hauptargument für die Ausübung einer Teilzeitbeschäftigung kaum an Bedeutung verloren hat.

Im Folgenden wird der Stand der Forschung bezüglich der Bestimmungsgründe der Teilzeitbeschäftigung skizziert. Auf die Beschreibung der Datengrundlage des SOEP und des methodischen Vorgehens folgt ein empirischer Überblick zur Entwicklung der Teilzeitbeschäftigung von 1991 bis 2006 nach Geschlecht sowie zum aktuellen Ausmaß der Teilzeit zusätzlich differenziert nach Altersgruppen. In einem nächsten Analyseschritt werden die Determinanten der Erwerbsübergänge von Frauen betrachtet, die eine Teilzeitbeschäftigung aufnehmen, und mit jenen kontrastiert, die eine Vollzeitstelle antreten, um zu beantworten, für welche Beschäftigtengruppen Teilzeit die Partizipation im Arbeitsmarkt erleichtert. Abschließend werden mögliche
Konsequenzen für die betroffenen Arbeitnehmerinnen sowie Arbeitnehmer diskutiert.

\section{Stand der Forschung}

Das Hauptmotiv um in Teilzeit erwerbstätig zu sein ist, dass diese Erwerbsform nach wie vor als Möglichkeit zur Vereinbarkeit von Familie und Beruf angesehen wird (vgl. Schmal 1997). Und in Anbetracht der Tatsache, dass die überwältigende Mehrheit der Teilzeitbeschäftigten weiblich ist, überrascht es nicht, dass eine ganze Reihe von Studien zum Thema auf die Besonderheiten der Erwerbssituation von Frauen bzw. von Müttern hinweist, von denen hier nur eine kleine Auswahl beispielhaft genannt werden soll (vgl. z. B. Quack 1993; Stephan 1995; Drobnic u. Wittig 1995; Blossfeld u. Hakim 1997; Tam 1997; O'Reilly u. Fagan 1998).

Tatsächlich sind mehr als die Hälfte der erwerbstätigen Mütter in Teilzeit oder geringfügiger Beschäftigung tätig, weil diese es entweder überhaupt erst ermöglicht oder aber erheblich erleichtert, familiäre und berufliche Verpflichtungen zu vereinbaren (vgl. Engstler u. Menning 2003). Besonders häufig wird die Teilzeitbeschäftigung von Müttern genutzt, um nach einer Familienphase wieder auf den Arbeitsmarkt zurückzukehren (vgl. z. B. Schupp 1991; Gustafsson et al. 1996; Büchel u. Spieß 2002). Zusätzlich kehren Mütter heute insgesamt wesentlich häufiger wieder ins Berufsleben zurück als noch die Generation ihrer eigenen Mütter. Blossfeld und Rohwer (1997) weisen diesen Kohorteneffekt auf Grundlage des SOEP nach: Für die Kohorten der ab 1934 und später geborenen Frauen zeigt sich - bereits kontrolliert für den Effekt der Ausweitung des Zugangs zu Bildungsmöglichkeiten - jeweils ein starker Anstieg der Wahrscheinlichkeit, aus dem Status der Hausfrau in eine Teilzeitbeschäftigung zu wechseln. Obwohl Blossfeld und Rohwer in ihrer Studie bereits ausdrücklich darauf hinweisen, dass sich die Erwerbsmuster in Ost- und Westdeutschland deutlich unterscheiden, bleibt ihre Analyse der Erwerbsübergänge von Teilzeitarbeitnehmerinnen jedoch auf Westdeutschland beschränkt.

Für die Zunahme der Zahl der Beschäftigten in Teilzeitarbeitsverhältnissen wird in der Literatur auf unterschiedliche Gründe verwiesen. Auf Arbeitgeberseite wird z. B. konstatiert, dass der steigende globale Wettbewerbsdruck zu einem erhöhten Bedarf an flexibel Beschäftigten führt, weil so Kosten eingespart werden können. Die Teilzeitbeschäftigung dient hier insbesondere dazu, die Arbeitszeitflexibilität in Unternehmen zu erhöhen, wodurch sich beispielsweise die Produktion kurzfristig an konjunkturelle Schwankungen anpassen oder Betriebs- und Öffnungszeiten kostengünstig verlängern lassen (vgl. z. B. Bruegel u. Hegewisch 1994).

Auf Seiten der Arbeitnehmer und Arbeitnehmerinnen ist ebenfalls der Wunsch nach Arbeitszeitflexibilität vorhan- 
den, um verschiedene Lebensbereiche besser vereinbaren $\mathrm{zu}$ können, wie etwa Beruf mit Weiterbildung oder eben mit Familie (vgl. z. B. Bothfeld 1997). Nicht unberücksichtigt bleiben darf allerdings, dass die individuellen Arbeitszeitpräferenzen nicht nur sehr heterogen sind, sondern die tatsächlich realisierbaren Erwerbsentscheidungen darüber hinaus auch von diesen jeweiligen Arbeitszeitpräferenzen mehr oder weniger stark abweichen.

Insgesamt ist eine Ausweitung der Teilzeitbeschäftigung zu erwarten, weil auf der einen Seite Arbeitgeber vermehrt Teilzeitkräfte nachfragen. Zunehmend werden beispielsweise Service- und Pflegekräfte sowie Positionen im öffentlichen Dienst in Teilzeit besetzt, und bereits heute ist eine große Mehrheit der Teilzeitstellen im Dienstleistungsbereich angesiedelt (vgl. z. B. Kohler u. Spitznagel 1995). Auf der anderen Seite fragen insbesondere Frauen, die aufgrund familiärer Verpflichtungen nicht in Vollzeit erwerbstätig sein können oder wollen, heute vermehrt Teilzeitstellen als Alternative zum Hausfrauenstatus nach (vgl. z. B. Blossfeld u. Rohwer 1997).

Allerdings sind beim beruflichen Wiedereinstieg gerade Teilzeitarbeitsverhältnisse mit Abstiegs- und Dequalifizierungsrisiken verbunden (vgl. etwa McRae 1991 für Großbritannien). Für erwerbstätige Frauen, die auf den Arbeitsmarkt zurückgekehrt sind, zeigt Waldfogel (1995) ebenfalls in einer britischen Studie, dass mit der Rückkehr in eine Teilzeitanstellung ein größerer Einkommensverlust einhergeht als in eine Vollzeitanstellung. Vergleichbare Befunde, nach denen sich beispielsweise mit zunehmender Dauer des Erziehungsurlaubs die weiteren Karrierechancen von Frauen verringern, liegen für Deutschland vor (vgl. Ziefle 2007). Ebenso gilt für den weiteren Erwerbsverlauf, dass Teilzeitbeschäftigte ein höheres Risiko aufweisen, im Falle eines beruflichen Wechsels abzusteigen als Vollzeitbeschäftigte; gleichzeitig haben sie eine geringere Chance, beruflich aufzusteigen (vgl. Schäfer u. Vogel 2005). Darüber hinaus sind Teilzeitbeschäftigungsverhältnisse generell von durchschnittlich geringerer Dauer (vgl. Vogel 2007).

In der Literatur bleibt die Bewertung der Teilzeitarbeit ambivalent, denn obwohl Teilzeit insbesondere Müttern zusätzliche Partizipationschancen am Arbeitsmarkt eröffnet, bleiben die beruflichen Perspektiven begrenzt. Offen ist, wie kontinuierlich die - im Schnitt geringeren - Teilhabechancen sind und ob bzw. unter welchen Bedingungen Teilzeiterwerbstätigkeit den Übergang in eine Vollzeitbeschäftigung erleichtern kann. Darüber hinaus mangelt es an belastbaren Studien über teilzeitbeschäftigte Männer, obwohl anzunehmen ist, dass sich die Chancen bzw. Risiken, die Teilzeitbeschäftigung mit sich bringt, für verschiedene Gruppen von Beschäftigten und insbesondere für Männer und Frauen deutlich unterscheiden. Um zu beschreiben, wer Teilzeit arbeitet, werden im Folgenden Teilzeitquoten nach Geschlecht und Altersgruppen präsentiert und die
Determinanten der Beschäftigungsübergänge von Frauen in Teilzeit und in Vollzeit vergleichend analysiert.

\section{Daten, Variablen und Methode}

Die Auswertungen basieren auf den Daten des SOEP der Jahre 1991 bis 2006, die sowohl den Querschnittvergleich über verschiedene Zeitpunkte als auch die Längsschnittanalyse von Erwerbsübergängen zulassen. Die Stichprobe umfasst für Westdeutschland knapp 13.000 und für Ostdeutschland etwa 4.500 Personen, die - jährlich wiederholt - unter anderem zu den Themen Beruf und Familie befragt werden (Anger et al. 2008).

Als in Teilzeit tätig werden im Folgenden Beschäftigte mit einer wöchentlichen Arbeitszeit von höchstens 35 Stunden betrachtet, die sich selbst auf die Frage nach ihrer derzeitigen Erwerbstätigkeit als in Teilzeit oder geringfügig beschäftigt einstufen. Die auf dieser Grundlage berechneten Teilzeitquoten geben den jeweiligen Anteil der in Teilzeit Beschäftigten an allen Beschäftigten wieder. Alle in diesem Artikel präsentierten deskriptiven Befunde basieren auf Querschnittanalysen der Personendaten aus den jeweils ausgewiesenen Jahren. Die Ergebnisse sind unter Berücksichtigung entsprechender Personengewichte berechnet, so dass sie auf die Bevölkerung der Bundesrepublik verallgemeinert werden können. Allerdings beziehen sich die Analysen jeweils ausschließlich auf Personen im erwerbsfähigen Alter zwischen 16 und 65 Jahren.

Die Erwerbsübergänge sind als Wechsel aus einem Erwerbszustand in einen nächsten anderen definiert. Im Einzelnen wird unterschieden zwischen Vollzeitbeschäftigten, Teilzeit- oder geringfügig Beschäftigten und Nichterwerbstätigen (ohne Arbeitslose). ${ }^{1}$ Um zu untersuchen, wer eine Teilzeit- bzw. wer eine Vollzeitbeschäftigung aufnimmt, ist zusätzlich jeweils relevant, aus welchem Erwerbsstatus heraus eine solche Beschäftigungsaufnahme erfolgt. Deshalb resultiert die Festlegung der vier hier zu analysierenden Erwerbsübergänge aus der Kombination von Ausgangs- und Zielerwerbszuständen: 1. aus Vollzeit in Teilzeit (VZ/TZ), 2. aus Teilzeit in Vollzeit (TZ/VZ), 3. aus Nichterwerbstätigkeit in Vollzeit (NE/VZ) und 4. aus Nichterwerbstätigkeit in Teilzeit (NE/TZ). Die vier abhängigen Übergangs- bzw. Ereignisvariablen nehmen jeweils den Wert 1 an, wenn der Erwerbsübergang aus dem entsprechenden Ausgangsstatus in den entsprechenden

\footnotetext{
${ }^{1}$ Die Determinanten der Erwerbsübergänge Arbeitsloser müssen aufgrund spezifischer Merkmale ihres Status, die dazu führen, dass die Entscheidungsmechanismen von jenen Nichterwerbstätiger systematisch abweichen, separat analysiert werden. Es kann in der Regel davon ausgegangen werden, dass Arbeitslose per Definition aktiv eine Stelle suchen und bereits Arbeitsmarkterfahrung mitbringen, was auf Nichterwerbstätige nicht zutreffen muss.
} 
Zielzustand erfolgt ist, sie nehmen jeweils den Wert 0 an, wenn kein solcher Übergang stattgefunden hat (zur Ereignisanalyse vgl. z. B. Allison 1984). Erwerbsepisoden, die nicht mit dem zu untersuchenden Zielzustand enden, werden als rechtszensiert betrachtet, und zwar unabhängig davon, ob sie mit dem Übergang in einen anderen alternativen Zielzustand enden oder aber weiter andauern. Das heißt im Falle der Rechtszensierung nimmt die Ereignisvariable jeweils den Wert 0 an.

Außerdem ist für jede Person bekannt, in welchem Monat ein Erwerbsübergang erfolgt ist bzw. wie viele Monate die Person im Ausgangszustand verweilt. Diese zusätzliche Information stammt aus den Kalenderdaten des SOEP, die jeweils den individuellen Erwerbszustand aus den Monaten zwischen zwei Befragungen detailliert enthalten. Aus den vereinten Informationen aus Personen- und Kalenderdaten wird eine Personen-Monats-Datenbasis erstellt, auf der für jeden Erwerbsübergang separat multivariate Modelle geschätzt werden. Mehrere Erwerbsübergänge einer Person gehen unabhängig voneinander in die Analyse ein.

Die im Folgenden präsentierten Ergebnisse basieren auf logistischen Regressionsmodellen (vgl. z. B. DeMaris 1992). In den einzelnen Modellen ist jeweils zusätzlich für die Dauer der Erwerbsphasen im Ausgangsstatus (mit den Ausprägungen 6, 12, 18, 24, 36, 48 Monate und mehr) kontrolliert, weil die Wahrscheinlichkeit eines Erwerbsübergangs mit der Verweildauer im Ausgangszustand variiert, ohne jedoch auf die Komplexität der Zeitabhängigkeit von Erwerbsübergängen im Folgenden weiter eingehen zu können. Darüber hinaus sind die Modelle aufgrund der jeweiligen Selektion der Risikogruppe nach Ausgangszustand nicht repräsentativ und sie werden ungewichtet berechnet. Der Tatsache, dass mehrere Beobachtungen pro Befragten vorliegen - aufgrund der Panelstruktur der Daten sowie der generierten Personen-Monats-Datenbasis - und diese offensichtlich nicht unabhängig voneinander sind, wird durch ein entsprechendes in STATA implementiertes Schätzverfahren für Paneldaten Rechnung getragen, das die Clusterstruktur der Daten kontrolliert und die Standardfehler anpasst.

In den multivariaten Übergangsanalysen werden im Einzelnen die Effekte der sozio-ökonomischen Korrelate wie etwa „Lebensalter“ und „Bildung“ sowie der familialen Determinanten wie „Familienstand“ und „Alter des jüngsten Kindes" auf die jeweiligen Erwerbsübergänge von Müttern untersucht. Die Interpretation der in den Modellen ausgewiesenen Odds Ratios erfolgt in Abhängigkeit von der jeweiligen Gesamtübergangsrate, d. h. von der Wahrscheinlichkeit, den in diesem Modell untersuchten Erwerbsübergang überhaupt zu machen. Technisch drücken Odds Ratios die Relation zweier Größenverhältnisse aus und können folgendermaßen gelesen werden: Ein Odds Ratio größer 1 (kleiner 1) besagt, dass die benannte Gruppe eine im Vergleich zur Referenzgruppe höhere (geringere) Wahrscheinlichkeit aufweist, den untersuchten Erwerbsübergang zu erfahren (vgl. z. B. Diaz-Bone 2006).

Die unabhängigen Variablen „Lebensalter“ sowie „Alter des jüngsten Kindes" sind zeitabhängig modelliert, damit das tatsächliche Alter zum Zeitpunkt des Erwerbsübergangs und nicht etwa das Alter bei Aufnahme des untersuchten Ausgangszustandes in die Regressionsschätzungen eingeht. Bildung ist dagegen zeitinvariant modelliert, weil angenommen wird, dass das einmal erreichte Bildungsniveau im Lebenslauf relativ stabil bleibt. Auch die Variable „Region“ ist zeitunabhängig modelliert, so dass ihr Einfluss in erster Linie als Sozialisationseffekt interpretiert werden kann, mit dem unterschiedliche normative Konzepte der geschlechtsspezifischen Arbeitsteilung in Ost- und Westdeutschland einhergehen. Aber auch strukturelle und institutionelle Unterschiede zwischen den alten und den neuen Bundesländern spiegeln sich wider. Am Beispiel Region kann die inhaltliche Interpretation der Odds Ratios veranschaulicht werden: Im Modell des Übergangs von Teilzeit in Vollzeit wird für Ostdeutschland ein Odds Ratio von 1,31 ausgewiesen. Dieser Wert besagt, dass ostdeutsche Frauen im Verhältnis zu westdeutschen Frauen um das 1,31-Fache häufiger in Vollzeit wechseln.

Aufgrund der Annahme, dass die Erwerbsentscheidungen von Männern und Frauen von jeweils verschiedenen Variablen abhängen und von teils gegenläufigen Effekten geprägt werden, sind die Analysen nach Geschlecht getrennt durchgeführt worden. Weil die Modelle für teilzeitbeschäftigte Männer aufgrund der geringen Fallzahlen jedoch nicht aussagekräftig sind, werden die Ergebnisse der Übergangsanalysen im Folgenden ausschließlich für Frauen präsentiert.

\section{Deskriptive Befunde zur Entwicklung der Teilzeitbeschäftigung}

Die Teilzeitquoten sind in der Bundesrepublik seit 1991 deutlich gestiegen. Den Daten des SOEP zufolge waren im Jahre 2006 rund 47\% der weiblichen (1991: 33\%) und knapp 10\% der männlichen Beschäftigten (1991: 3\%) in Teilzeit tätig (siehe Abb. 1). Dabei ist Teilzeiterwerbstätigkeit bislang eine Domäne der Frauen geblieben: 2006 sind vier von fünf Teilzeitbeschäftigten weiblich. Der Anteil der weiblichen an allen Teilzeitbeschäftigten ist im betrachteten Zeitraum trotz einer Verdreifachung der Männerteilzeitquote nur leicht zurückgegangen, weil der Anstieg bei den männlichen Teilzeitbeschäftigten von einem sehr niedrigen Niveau ausgeht.

Ein Teilzeiterwerb wird vor allem deshalb überwiegend von Frauen ausgeübt, weil sie in weiten Teilen der Bevölkerung als die attraktivste Alternative erscheint, um berufliche und familiäre Verpflichtungen zu vereinbaren. Diese Vereinbarkeitsproblematik entsteht durch die den Frauen per 
Abb. 1 Entwicklung der Teilzeitquote - 1991 bis 2006 (nach Geschlecht)

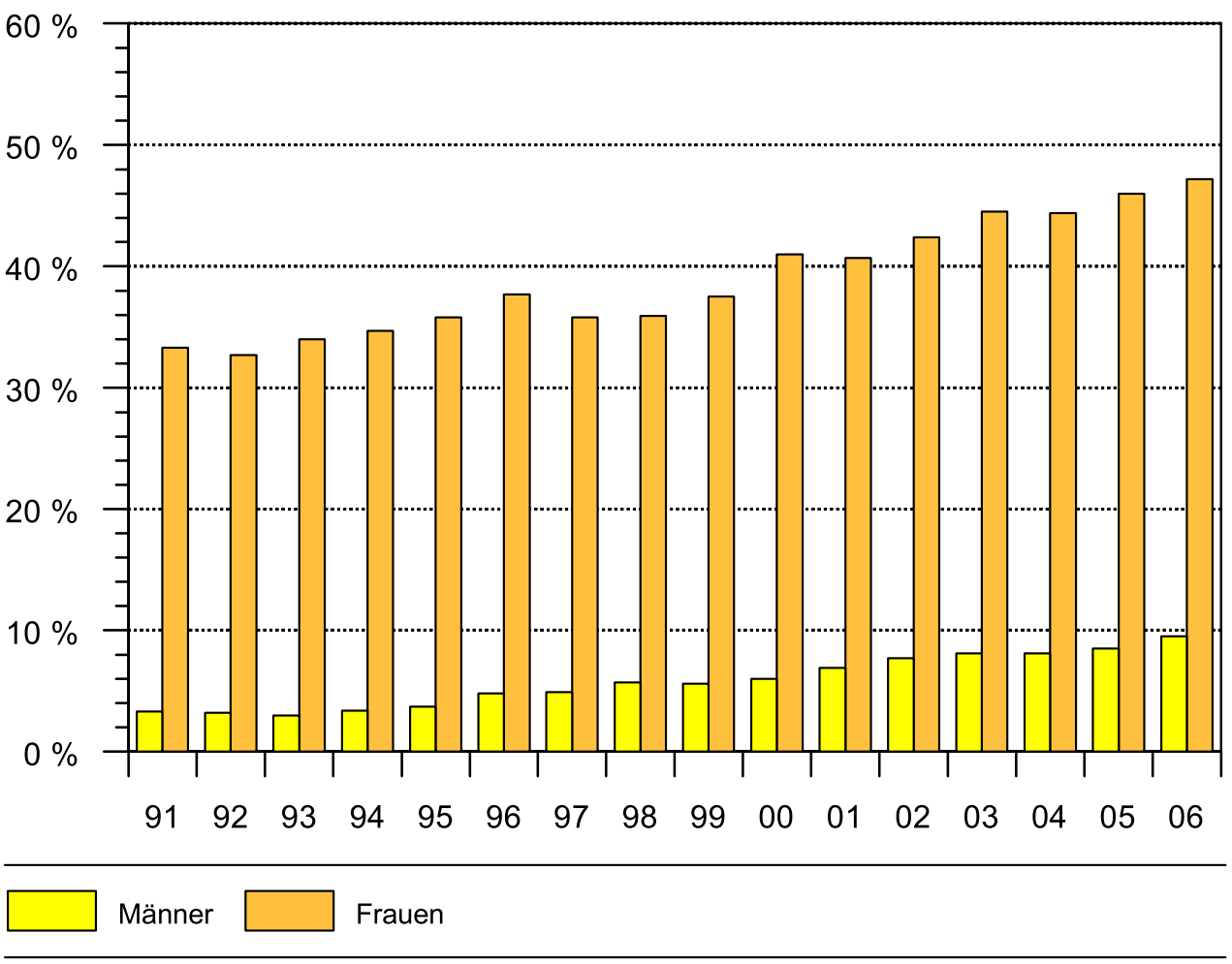

Quelle: SOEP 1991 bis 2006; eigene Berechnungen, gewichtet. traditioneller Arbeitsteilung zugedachte Rolle als Familienverantwortliche, die die Hauptlast der Haus- und Betreuungsarbeit leistet und höchstens einen Zuverdienst zum Familieneinkommen beisteuert (vgl. z. B. Holst u. Maier 1998). Für Männer und insbesondere für Väter besteht die Vereinbarkeitsproblematik bezüglich Berufstätigkeit und Familie in der Regel nicht, weil ihnen die Rolle des vollerwerbstätigen Versorgers zugewiesen wird, und zwar im normativen Konstrukt sowohl des traditionellen männlichen Ernährermodells als auch eines modernisierten Doppelverdiener-Ernährermodells.

Obwohl in letzterem die Rolle des Versorgers durch die Rolle des fürsorglichen Ehemanns und Vaters mit Familienpflichten erweitert wird - und entsprechend die Rolle der Ehefrau und Mutter um die Rolle der teilzeitbeschäftigten Zuverdienerin ergänzt ist - ändert dies meist nichts an der Entscheidung für den Vollzeiterwerb bei Männern. Deutlich sichtbar wird dies beispielsweise auch an der geringen Zahl von Vätern, die Elternzeit in Anspruch nehmen. Zusätzlich fragen männliche Erwerbstätige kaum Teilzeitarbeit nach, weil Arbeitgeber selten Teilzeitstellen anbieten, die entsprechend attraktiv ausgestattet sind, also beispielsweise ein ausreichendes hohes Einkommen einbringen, um eine Familie dauerhaft ernähren zu können. Für Frauen trägt die Modernisierung des normativen Ernährermodells hingegen zu einem weiteren Anstieg der Teilzeitquoten bei, denn heute gehen deutlich mehr Frauen einem eigenen Erwerb nach als dies beispielsweise noch in der Generation ihrer eigenen Mütter der Fall war.

Aufgrund der normativ verankerten geschlechtsspezifischen Arbeitsteilung im gesamten Lebenslauf unterscheiden sich die Befunde zum Teilzeiterwerb insbesondere nach Lebensalter für Männer und Frauen (siehe Abb. 2): Überdurchschnittlich ist die Teilzeitquote der Frauen in der Altersgruppe zwischen 36 und 45 Jahren. Mehr als die Hälfte dieser berufstätigen Frauen sind in Teilzeit tätig $(56 \%)$. Da die Familiengründung für diese Altersgruppe in der Regel bereits erfolgt und in vielen Fällen abgeschlossen ist, lässt sich vermuten, dass ein Großteil dieser Frauen eine Teilzeittätigkeit angenommen hat, um nach einer etwa aufgrund einer Familienphase erfolgten - Erwerbsunterbrechung wieder auf dem Arbeitsmarkt Fuß zu fassen. Darüber hinaus erleichtert die Teilzeitstelle nicht nur den Wiedereinstieg oder ermöglicht diesen erst, weil sich die Betreuung von noch kleinen Kindern und die Erwerbsarbeit bei diesem Arrangement nicht ausschließen, sondern sie erleichtert möglicherweise auch einen späteren Wechsel auf eine Vollzeitstelle und somit eine dauerhafte Partizipation im Arbeitsmarkt.

Die höchste Teilzeitquote weisen allerdings die 56- bis 65-jährigen Arbeitnehmerinnen auf: 59\% von ihnen sind Teilzeitbeschäftigte. Dieser Befund könnte zum einen auf einen Kohorteneffekt hinweisen, in dem sich die Modernisierung des männlichen Ernährermodells bereits teilweise 
Abb. 2 Teilzeitquote nach Altersgruppen - 2006 (nach Geschlecht)

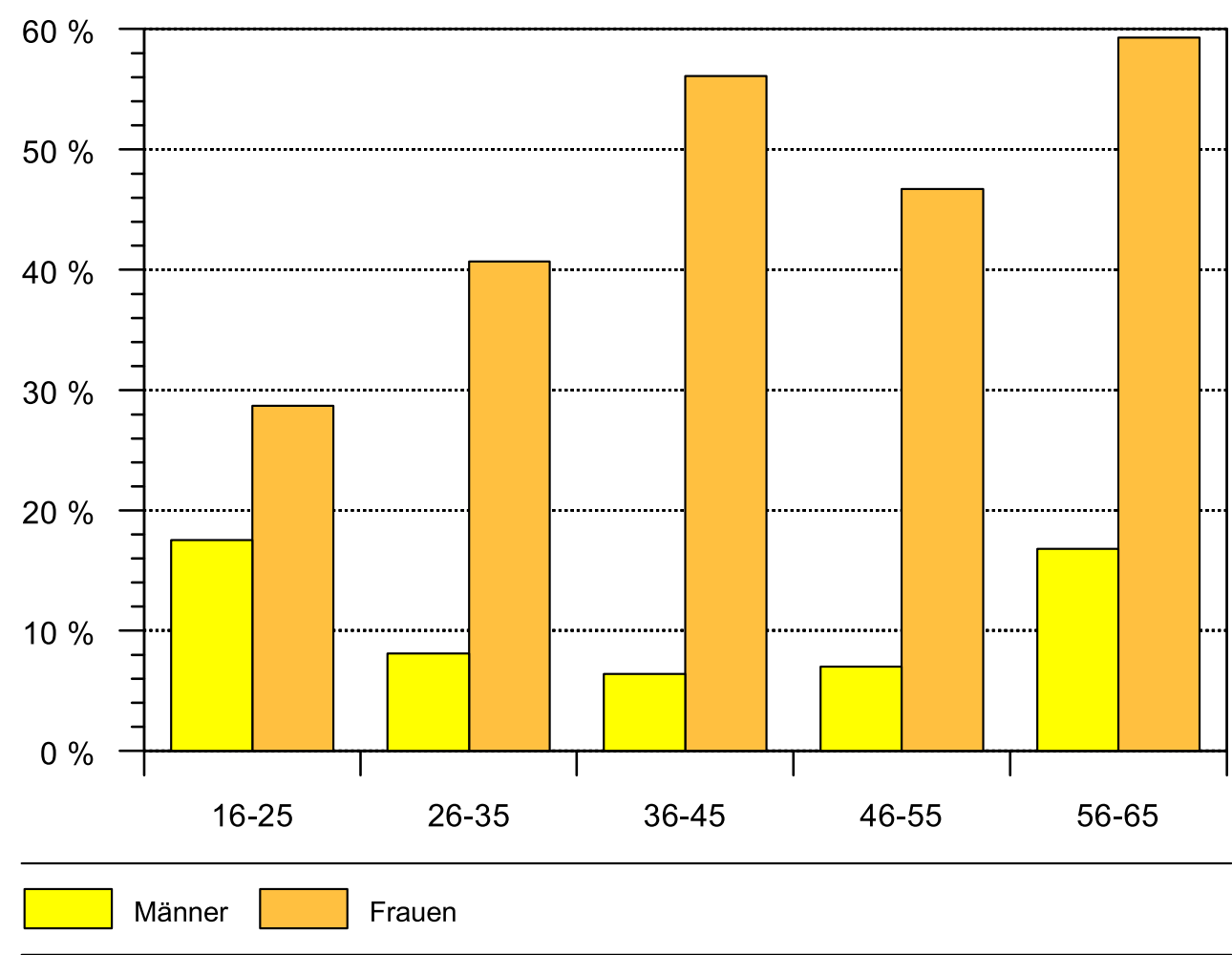

Quelle: SOEP 2006; eigene Berechnungen, gewichtet. spiegelt. Allerdings ist ein solcher Effekt in einer Querschnittsbetrachtung nicht eindeutig zu identifizieren, dies wäre erst in einem Vergleich mehrerer Querschnitte möglich. Der in Abb. 2 vorliegende Querschnittsbefund verweist zum anderen auf einen potenziellen Alterseffekt, der zumindest bei der ältesten hier betrachteten Altersgruppe den Kohorteneffekt überwiegen könnte: Zwar ist ein Teil dieser Frauen höchstwahrscheinlich bereits teilzeitbeschäftigt, seit ihre Kinder in den Kindergarten oder die Schule kamen, möglicherweise hat eine Gruppe der Teilzeitbeschäftigten ihre Stelle aber auch erst zu einem späteren Zeitpunkt angenommen, etwa nachdem die inzwischen erwachsenen Kinder bereits aus dem elterlichen Haushalt ausgezogen waren. Auf der Ebene der bivariaten Betrachtung sind zudem alternative Erklärungsansätze nicht auszuschließen, zu der für diese Altersgruppe etwa die Aufnahme einer Teilzeittätigkeit um Rentenanwartschaften zu erwerben bzw. eine etwaige gesetzliche Erwerbsminderungsrente aufzubessern zählen.

Auch die Teilzeitquote der 16- bis 25-jährigen Frauen, die zwar unter dem Durchschnitt liegt, aber mit $29 \%$ bereits eine substanzielle Höhe erreicht hat, verlangt nach alternativen Erklärungsansätzen, denn von der klassischen Vereinbarkeitsproblematik ist in dieser Altersgruppe offensichtlich nur ein kleinerer Teil betroffen. Hier scheint vielmehr die gesteigerte Nachfrage nach flexibel Beschäftigten durch die Arbeitgeberseite zu greifen. Übrigens fällt bei Männern die
Teilzeitquote der 16- bis 25 -Jährigen mit $18 \%$ aus denselben Gründen am höchsten aus (siehe Abb. 2); dicht gefolgt von der Teilzeitquote unter den 56- bis 65-jährigen Arbeitnehmern (17\%). Männliche Beschäftigte zwischen 26 und 55 Jahren sind dagegen am seltensten in Teilzeit tätig, die Quoten liegen zwischen nur 6 und 8\%, wie aufgrund des normativen Konstrukts des männlichen Ernährermodells auch in seinen modernisierten Varianten - zu erwarten war.

Bei beiden Gruppen, den älteren Arbeitnehmerinnen und den älteren Arbeitnehmern, ist aber auch ein Teil der Teilzeitbeschäftigung faktisch Vollzeiterwerbstätigkeit oder vorgezogener Ruhestand: Da die „Altersteilzeit“ meist in Form der Blockteilzeit ausgestaltet wird, scheiden die betroffenen Beschäftigten nach der in Vollzeit abgeleisteten ersten Hälfte der Altersteilzeit faktisch aus der Erwerbsarbeit aus (vgl. z. B. Lindecke et al. 2007). Obgleich ein flexibler Übergang in den Ruhestand wissenschaftlich und sozialpolitisch seit langem propagiert wird, und dies auch helfen könnte, Privates und Berufliches zu vereinbaren oder auch den Erfahrungstransfer am Arbeitsplatz zu gewährleisten, ist er bislang eine seltene Ausnahme. Und in Befragungen führen selbst die Teilzeitbeschäftigten über 55 Jahren praktisch nie das Motiv an, sich auf den Ruhestand vorbereiten zu wollen - auch in dieser Altersgruppe werden in erster Linie die Doppelbelastung durch Beruf und Haushalt sowie familiäre, gesundheitliche und betriebliche Gründe für die Teilzeiterwerbstätigkeit genannt (Künemund 
2000, S. 294). Allerdings besteht für diese Altersgruppe die Vereinbarkeitsproblematik möglicherweise darin, die eigene Erwerbstätigkeit auf die Pflege von Angehörigen abzustimmen.

Welche Konsequenzen Teilzeitbeschäftigung für die jüngeren Beschäftigten - mit Quoten von wie gezeigt knapp einem Fünftel der Männer und über einem Viertel der Frauen - haben wird, ist bislang kaum abzuschätzen, da diese den überwiegenden Teil des Erwerbslebens noch vor sich haben. Dabei ist insbesondere die Zahl der Berufseinsteiger, die als erste Stelle ein Teilzeitarbeitsverhältnis antreten, seit der Wiedervereinigung deutlich angestiegen. Aber nur, wenn nach dem direkt im Anschluss an die Berufsausbildung oder das Studium erfolgten Berufseinstieg in Teilzeit der Übergang in eine Vollzeitbeschäftigung gelingt, werden sich die durchschnittlich geringeren Einkommens- und Karrieremöglichkeiten in Teilzeit nicht zu einem dauerhaften Nachteil kumulieren. Prägen Teilzeitarbeitsverhältnisse, die auch mehr als doppelt so häufig befristet sind wie Vollzeitarbeitsverhältnisse (vgl. Giesecke 2006) hingegen dauerhaft den Erwerbsverlauf, könnte die Teilzeitbeschäftigung unter den gegebenen Arbeitsmarktbedingungen nicht nur kurzfristig, sondern auch langfristig zu Benachteiligungen führen, wie beispielsweise einer schlechteren individuellen
Absicherung im Alter. Die im folgenden Abschnitt präsentierten Ergebnisse der Analyse der Erwerbsübergänge von Frauen geben erste Hinweise, welche Bestimmungsgründe den Einstieg in die Teilzeit sowie den Übergang von einer Teilzeit- in eine Vollzeitstelle begünstigen.

\section{Determinanten der Erwerbsübergänge von Frauen}

Da die im Vergleich zur Vollzeit vermeintlich bessere Vereinbarkeit von Familie und Beruf als Hauptargument für die Aufnahme einer Teilzeitbeschäftigung durch weibliche Beschäftigte kaum an Bedeutung verloren hat, werden im Folgenden die familialen Bestimmungsgründe der Aufnahme einer Teilzeitbeschäftigung und der Aufnahme einer Vollzeitbeschäftigung vergleichend in den Blick genommen. Die in Tabelle 1 präsentierten Ergebnisse logistischer Regressionsmodelle ermöglichen den Vergleich des Einflusses der familialen Variablen „Familienstand“ und „Alter des jüngsten Kindes" als Determinanten der Aufnahme einer Teilzeit- bzw. einer Vollzeitbeschäftigung, und zwar unter Berücksichtigung des vorherigen Erwerbszustandes. Im Einzelnen werden die Bestimmungsgründe für vier Erwerbsübergänge separat analysiert: aus Nichterwerbstätig-
Tabelle 1 Determinanten der Erwerbsübergänge in Teilzeit und in Vollzeit (Frauen)

\begin{tabular}{lcccc}
\hline $\begin{array}{l}\text { Logistische Regressionen (Odds Ratios) } \\
\text { Separate Modelle }\end{array}$ & NE/VZ & NE/TZ & VZ/TZ & TZ/VZ \\
\hline Alter (16-25 Jahre) & & & & \\
$26-35$ & 0,856 & $1,355^{* *}$ & $1,635^{* *}$ & 1,062 \\
$36-45$ & $0,766^{*}$ & $1,447^{* *}$ & $1,884^{* *}$ & 0,999 \\
$46-55$ & $0,402^{* *}$ & 1,139 & $2,037^{* *}$ & $0,749^{* *}$ \\
56-65 & $0,011^{* *}$ & $0,449^{* *}$ & $2,221^{* *}$ & $0,233^{* *}$ \\
Alter des jüngsten Kindes in Jahren (Keine Kinder) & & & \\
0-3 & 0,978 & $2,542^{* *}$ & $1,304^{*}$ & $0,376^{* *}$ \\
4-6 & 0,789 & $1,976^{* *}$ & $1,440^{* *}$ & $0,503^{* *}$ \\
7-15 & 0,955 & $1,507^{* *}$ & $1,254^{* *}$ & $0,787^{* *}$ \\
Familienstand (Nie verheiratet) & & & & \\
Verheiratet & $0,683^{* *}$ & $1,358^{* *}$ & $1,510^{* *}$ & 0,906 \\
Geschieden & 0,929 & 1,035 & $1,224^{*}$ & $1,394^{* *}$ \\
Verwitwet & 0,485 & 0,886 & $1,443^{*}$ & 1,166 \\
Bildungsniveau (Niedrig) & & & & \\
Mittel & 0,917 & 1,060 & $1,277^{* *}$ & 0,918 \\
Hoch & $1,325^{*}$ & 1,083 & $1,401^{* *}$ & $1,286^{* *}$ \\
Region (West) & & & \\
Ost & 0,807 & $0,499^{* *}$ & $0,656^{* *}$ & $1,308^{* *}$ \\
Interaktion (Alter des jüngsten Kindes* Region) & & & \\
0-3* Ost & $2,852^{* *}$ & 1,233 & 0,921 & $2,541^{* *}$ \\
4-6* Ost & $2,421^{*}$ & 0,987 & 0,817 & $1,417^{*}$ \\
7-15* Ost & 1,289 & 0,760 & 0,927 & $1,457^{* *}$ \\
Fallzahl (Personenmonate) & 206.458 & 206.458 & 333.213 & 239.604 \\
Pseudo R & 0,19 & 0,19 & 0,18 & 0,14 \\
\hline
\end{tabular}

Quelle: SOEP 1991 bis 2006 ; eigene Berechnungen. ${ }^{*} p<0,05 ;{ }^{* *} p<0,01$.

${ }^{1}$ Zusätzlich wurde in jedem Modell kontrolliert für die Dauer der Beschäftigung (in Monaten) im Ausgangszustand (nicht in der Tabelle ausgewiesen) 
keit in Vollzeit (NE/VZ), aus Nichterwerbstätigkeit in Teilzeit (NE/TZ), aus Vollzeit in Teilzeit (VZ/TZ), sowie aus Teilzeit in Vollzeit (TZ/VZ). Die Ergebnisse der vier Modelle sind in Tabelle 1 dargestellt.

Tatsächlich lassen sich sowohl Mutterschaft wie auch Ehe als relevante Bestimmungsgrößen identifizieren, die bei Frauen für die Aufnahme einer Teilzeitarbeit sprechen. Mütter, die ihre Erwerbstätigkeit unterbrochen haben, d. h. zumindest zeitweilig nichterwerbstätig waren, weisen im Vergleich zur Referenzgruppe nichterwerbstätiger kinderloser Frauen signifikant höhere Wahrscheinlichkeiten auf, eine Teilzeitstelle anzutreten (siehe Tabelle 1: Modell NE/TZ). Für Mütter von Kleinkindern im Alter zwischen 0 und 3 Jahren beträgt das Odds Ratio 2,54. Und für Mütter von Kindern, die mindestens im Alter zwischen 7 und 15 Jahren sind, beträgt das Odds Ratio für den Übergang in die Teilzeit noch 1,51. Das bedeutet, in der selektiven Risikogruppe der nichterwerbstätigen Frauen haben Mütter eine größere Chance, tatsächlich eine Teilzeitstelle aufzunehmen. Dies erscheint plausibel, weil die Geburt eines Kindes ein gewichtiger Grund ist, die Erwerbskarriere überhaupt erst zu unterbrechen und zeitweise nichterwerbstätig zu sein. Die vergleichsweise geringere Teilzeitübergangsrate der kinderlosen Frauen, die aus anderen Gründen nichterwerbstätig sind, könnte darauf zurückzuführen sein, dass sie dem Arbeitsmarkt generell nicht mehr zur Verfügung stehen, etwa aufgrund von gesundheitlichen Einschränkungen bis hin zur Erwerbsunfähigkeit oder einfach, weil sie dauerhaft Hausfrau bleiben. Solche Frauen hingegen, die aktiv auf der Suche nach einer Arbeitsstelle sind, würden sich möglicherweise eher arbeitslos melden. Arbeitslose aber sind wie erläutert in der vorliegenden Studie nicht in der Gruppe der Nichterwerbstätigen enthalten.

Gleichzeitig reduzieren bislang vollzeitbeschäftigte Mütter ihre Arbeitszeit signifikant häufiger als in Vollzeit beschäftigte kinderlose Frauen, wobei es sich auch bei den vollbeschäftigten Frauen wiederum um eine selektive Risikogruppe handelt (siehe Tabelle 1: Modell VZ/TZ): Selbst bei Müttern mit einem jüngsten Kind im schulpflichtigen Alter beträgt das Odds Ratio des Übergangs in die Teilzeit 1,25 , bei Müttern mit Kindern im Kindergartenalter beträgt es 1,44 und bei Müttern von Kleinkindern im Alter zwischen 0 und 3 Jahren beträgt es 1,30. Darüber hinaus nehmen verheiratete Frauen, die zuvor nicht erwerbstätig waren und solche, die zuvor vollerwerbstätig waren, eher eine Teilzeitbeschäftigung auf als ledige - jeweils bereits kontrolliert für Mutterschaft und alle weiteren Kovariate, die in die Gesamtmodelle eingehen. Die Odds Ratios der Verheirateten betragen 1,36 (siehe Tabelle 1: Modell NE/TZ) bzw. 1,51 (siehe Tabelle 1: Modell VZ/TZ).

Umgekehrt sind die Bestimmungsgründe, die für die Aufnahme einer Vollzeittätigkeit sprechen, bei Frauen Ehelosigkeit (signifikant beim Übergang Nichterwerbstä-
tigkeit/Vollzeit) bzw. Scheidung sowie Kinderlosigkeit (signifikant beim Übergang aus Teilzeit in Vollzeit). Darüber hinaus ist die Aufnahme einer Vollzeitbeschäftigung bei Frauen in erster Linie abhängig von aus der Literatur bekannten Humankapitaleffekten. Die Chance auf einen Wechsel in die Vollzeit ist nicht nur vergleichsweise am größten für die jüngsten Beschäftigten - und am kleinsten für die ältesten Arbeitnehmerinnen zwischen 56 und 65 Jahren (siehe Tabelle 1: Modell TZ/VZ: Odds Ratio von $0,23)$-, sondern auch am größten für die Frauen mit der höchsten Bildung (siehe Tabelle 1: Modell TZ/VZ: Odds Ratio von 1,29).

Zusätzlich wird in den Regressionsmodellen nach regionalen Unterschieden zwischen Ost- und Westdeutschland kontrolliert. Dies geschieht u. a. aufgrund regionaler Besonderheiten der Arbeitsmärkte - beispielsweise ist der ostdeutsche Arbeitsmarkt nach der Wiedervereinigung durch gravierende Strukturveränderungen geprägt. In dieser Studie interessiert aber insbesondere der Einfluss unterschiedlicher normativer Einstellungen zur Vereinbarkeit von Familie und Beruf in West- und Ostdeutschland. Während in Westdeutschland die traditionelle geschlechtsspezifische Arbeitsteilung mehr Befürworter findet, sind Ostdeutsche eher positiv gegenüber der Erwerbstätigkeit von Müttern eingestellt und weisen im Durchschnitt modernere Einstellungen bezüglich der geschlechtsspezifischen $\mathrm{Ar}$ beitsteilung auf (vgl. z. B. Scheuer u. Dittmann 2007). In den Ergebnissen zeigen sich in der Tat die zu erwartenden regionalen Unterschiede im Vergleich von Frauen aus Ostund aus Westdeutschland. Ostdeutsche Frauen nehmen nach einer Erwerbsunterbrechung deutlich seltener eine Teilzeitstelle an (siehe Tabelle 1: Modell NE/TZ: Odds Ratio von 0,50). Außerdem gelingt ostdeutschen Frauen im Verhältnis zu westdeutschen signifikant häufiger der Übergang aus Teilzeit in Vollzeit (siehe Tabelle 1: Modell TZ/VZ: Odds Ratio von 1,31).

Aber ostdeutsche Frauen arbeiten nicht nur insgesamt seltener Teilzeit als westdeutsche, sondern auch dann, wenn sie Kinder haben. Umgekehrt wechseln ostdeutsche Mütter häufiger in Vollzeit. Beim Übergang aus einer Erwerbsunterbrechung in eine Vollzeitbeschäftigung (siehe Tabelle 1: Modell NE/VZ) beläuft sich der Interaktionseffekt der Merkmalskombination ostdeutsche Mutter mit mindestens einem Kind im Alter von unter vier Jahren auf ein Odds Ratio von 2,85. Das heißt diese ostdeutschen Mütter nehmen fast dreimal häufiger eine Vollzeitstelle auf als vergleichbare westdeutsche Mütter. Ein ähnliches Muster zeigt sich für den Übergang aus Teilzeit in Vollzeit, auch dieser ist für ostdeutsche Mütter vergleichsweise wahrscheinlicher. Für diese ostdeutschen Frauen und insbesondere Mütter übernehmen Teilzeitstellen somit häufiger die Funktion als Sprungbrett in eine Vollzeitanstellung. Für diese Interpretation spricht auch, dass die durchschnittliche 
Verweildauer in Teilzeitbeschäftigungsverhältnissen bei ostdeutschen Frauen geringer ist als bei westdeutschen. Frauen in Westdeutschland verbleiben dagegen dauerhafter in Teilzeitpositionen (vgl. Bothfeld u. O'Reilly 2000; Schäfer u. Vogel 2005).

Offensichtlich kann Teilzeiterwerbstätigkeit der besseren Vereinbarkeit von Familie und Beruf dienen. Gelebt wird dieses Arrangement aber eher von Frauen und Müttern in Westdeutschland, denn dort sind die traditionelle geschlechtsspezifische Arbeitsteilung und das männliche Ernährermodell noch weiter verbreitet. In Ostdeutschland wird die Vereinbarkeit von Familie und Beruf hingegen über andere Arrangements realisiert, und beispielsweise durch ein weites Netz an öffentlichen und ganztägig geöffneten Kinderbetreuungseinrichtungen auch für Kinder unter drei Jahren unterstützt (vgl. z. B. Hank et al. 2004). Teilzeitbeschäftigung und damit eine Reduzierung der Erwerbstätigkeit von Müttern spielt in Ostdeutschland eine untergeordnete Rolle.

\section{Diskussion}

Teilzeitbeschäftigung mit ihren Vorteilen - z. B. Arbeitszeitflexibilität und mehr Zeit außerhalb der Erwerbsarbeit etwa für Familie - und ihren Nachteilen, wie etwa aufgrund der kürzeren Arbeitszeiten geringeren Einkommen, ist nicht für alle Erwerbstätigen in gleichem Maße attraktiv. Obwohl auch die Teilzeitquoten der männlichen Beschäftigten gestiegen sind - fast jeder zehnte Arbeitnehmer ist teilzeitbeschäftigt - stellen Frauen die überwiegende Mehrheit der Teilzeitbeschäftigten: vier von fünf Teilzeitbeschäftigten sind weiblich. Überdurchschnittlich häufig arbeiten Frauen über 35 Jahren in Teilzeit und insbesondere ältere Arbeitnehmerinnen sowie auch ältere Arbeitnehmer zwischen 56 und 65 Jahren. Als relevante Determinanten der Erwerbsübergänge in Teilzeitbeschäftigung zeigen sich in erster Linie familiale Bestimmungsgründe: Sowohl Mutterschaft als auch Ehe sprechen bei Frauen für die Aufnahme einer Teilzeitstelle. Teilzeitarbeit wird vermehrt von Frauen und insbesondere Müttern ausgeübt, um verschiedene zentrale Lebensbereiche - in der Regel Familie und Beruf - zumindest zeitweise miteinander zu vereinbaren. Die Teilzeit ermöglicht also insbesondere jenen Arbeitsmarktgruppen die Erwerbstätigkeit, die aufgrund von Verpflichtungen in anderen Lebensbereichen nicht in Vollzeit partizipieren können oder wollen. Dabei eröffnet sie den Beschäftigten durchaus individuelle, über den Beruf vermittelte materielle und soziale Teilhabechancen. Ein eigenes Einkommen, individuelle Beiträge zu Arbeitslosen-, Kranken- und Rentenversicherung sowie soziale Integration über berufliche Kontakte sind gewährleistet. Darüber hinaus ist die Teilzeitbeschäftigung - insbesondere für jüngere sowie für ostdeutsche Arbeitnehmerinnen - häufig ein Sprungbrett in eine Vollzeitanstellung.

Folglich erscheint der Gedanke nahe liegend, das Potenzial der Teilzeitbeschäftigung könnte auch durch andere Arbeitsmarktgruppen zukünftig noch stärker genutzt werden, um die Belastung durch Erwerbsarbeit im Lebensverlauf umzuverteilen. Dadurch könnten Phasen der Ausbildung, der Familiengründung oder auch der Pflegearbeit erheblich entlastet oder Phasen der beruflichen Weiterentwicklung leichter eingeflochten werden. In solchen Situationen könnte Teilzeitarbeit eine Alternative auf Zeit zur Vollzeitbeschäftigung darstellen, und zwar für Frauen und Männer. Auch aufgrund der steigenden Lebenserwartung erscheint eine Umverteilung von bezahlter und unbezahlter Arbeit im Lebensverlauf sinnvoll. Beispielsweise kann Erwerbsarbeit mit Hilfe von Teilzeitbeschäftigung auf eine längere Lebensspanne umverteilt werden, die das bisherige Renteneintrittsalter von 65 bzw. zukünftig 67 Jahren möglicherweise weit überschreitet (vgl. Vaupel u. Loichinger 2006). Die entscheidende Frage bleibt aber, ob die durch Teilzeitbeschäftigung ermöglichten sozialen und materiellen Teilhabechancen ausreichend sind bzw. wie Teilzeitarbeitsverhältnisse ausgestaltet werden müssten, um diese zu gewährleisten ohne gleichzeitig bestehende soziale Ungleichheiten bspw. zwischen den Geschlechtern zu verschärfen.

Die individuellen Partizipationschancen der Teilzeitbeschäftigten lassen sich jedoch nur verbessern, indem die angebotenen Teilzeitstellen bezüglich Einkommen, Qualifikationsniveau und Karrierechancen zumindest ähnlich wie Vollzeitstellen ausgestattet werden. Dies ist bislang nur in Ausnahmefällen der Fall, vornehmlich im öffentlichen Dienst. Insbesondere für Berufseinsteiger, bei denen sich die zeitweise Schlechterstellung durch Teilzeitphasen über den gesamten Berufsverlauf kumulieren könnte, ist die Ausgestaltung der Teilzeitarbeitsverhältnisse von größter Bedeutung. Zusammengefasst lässt sich festhalten, dass das Potenzial, individuelle Partizipationschancen zu vermitteln, bislang nicht vollständig genutzt wird, weil die Rahmenbedingungen der Teilzeit nicht angemessen angepasst sind. Ein Bedarf an attraktiven Teilzeitstellen auf dem Level qualifizierter Beschäftigung besteht ebenso fort wie ein entsprechend ausgeweitetes Angebot an Kinderbetreuungseinrichtungen, um die Vereinbarkeit von Familie und Berufstätigkeit zu erleichtern.

Was die Zunahme der Teilzeit für die soziale Ungleichheit bedeutet, kann hier nur ansatzweise diskutiert werden. Bezüglich der geschlechtsspezifischen Ungleichheit auf dem Arbeitsmarkt deuten die für die Teilzeitbeschäftigung vorliegenden Befunde auf eine Egalisierung insbesondere bei den jüngeren Beschäftigtengruppen hin, weil sich die geschlechtsspezifischen Partizipationsmuster und Teilzeitquoten angleichen. Allerdings kann diese Annäherung nicht 
über das Fortbestehen geschlechtspezifischer Ungleichheiten im Arbeitsmarkt und darüber hinaus hinwegtäuschen, weil sich die Erwerbsmuster von Männern und Frauen besonders in den späteren Phasen des Erwerbsverlaufs unterscheiden und weiter voneinander entfernen, in denen sowohl zentrale berufliche Entscheidungen für die weitere Karriere als auch zentrale Entscheidungen bezüglich der Familiengründung fallen. Zudem ist die Verringerung der Geschlechterunterschiede hauptsächlich durch eine Heterogenisierung des Erwerbsverhaltens getrieben: Zwar sind Frauen häufiger als beispielsweise noch ihre eigenen Mütter erwerbstätig, aber Männer stehen auch seltener als beispielsweise ihre Väter im Normalarbeitsverhältnis. Insgesamt geht durch die Heterogenisierung der Erwerbsformen mit der Flexibilisierung von Beschäftigung auch eine größere Streuung von Teilhabechancen einher. Zusammengenommen weisen diese Argumente darauf hin, dass die Ungleichheit zwischen den Geschlechtern auf dem Arbeitsmarkt zwar abnimmt, bedingt durch die Heterogenisierung sowohl der weiblichen als auch der männlichen Beschäftigten aber eine Zunahme der sozialen Ungleichheit im Hinblick auf die realisierten Teilhabechancen zu erwarten ist. Sicherlich besteht weiterer Forschungsbedarf bezüglich der Erwerbsverläufe, die etwa mit Verfahren der Sequenzanalyse in ihrer Gesamtheit untersucht werden müssten sowie insbesondere bezüglich der Konsequenzen von Teilzeitbeschäftigung für die soziale Ungleichheit zwischen unterschiedlichen Gruppen von Arbeitnehmerinnen und Arbeitnehmern.

\section{Kurzfassung}

Eine dauerhafte Vollzeitbeschäftigung ermöglicht Arbeitnehmerinnen und Arbeitnehmern soziale und materielle Partizipationschancen. Dies gilt sowohl für die Sicherung des aktuellen Lebensstandards als auch im Hinblick auf zukünftige Perspektiven, etwa aufgrund erworbener Ansprüche in der gesetzlichen Rentenversicherung oder durch die Möglichkeiten zur privaten Altersvorsorge. Empirisch überwiegt in der Bundesrepublik bislang die in der Regel unter dem Begriff des Normalarbeitsverhältnisses gefasste kontinuierliche Vollzeitbeschäftigung. Allerdings ist zum Ende des 20. Jahrhunderts, spätestens seit der Wiedervereinigung eine stetige Zunahme der Teilzeitbeschäftigung zu beobachten. Laut Statistischem Bundesamt stieg die Zahl der Teilzeitbeschäftigten zwischen 1991 und 2004 von 4,7 auf 7,2 Millionen. Das entspricht bereits fast einem Viertel aller Beschäftigten (23\%), während dieser Anteil 1991 noch bei $14 \%$ lag.

Da Teilzeitarbeit sicherlich nicht für alle Arbeitsmarktgruppen in gleichem Maße attraktiv ist, untersucht der vorliegende Beitrag, in welchem Umfang Teilzeit von Frauen und Männern ausgeübt wird, wie die Beteiligung nach Al- tersgruppen variiert und welche Merkmale die Aufnahme einer Teilzeit- bzw. Vollzeitbeschäftigung bei Frauen begünstigen. Der Fokus der Betrachtung liegt auf den familialen Determinanten der Übergänge, da die Vereinbarkeit von Familie und Beruf als Hauptargument für die Ausübung einer Teilzeitbeschäftigung kaum an Bedeutung verloren hat.

Die Auswertungen basieren auf den Daten des Sozioökonomischen Panels (SOEP) der Jahre 1991 bis 2006, die sowohl den Querschnittvergleich über verschiedene Zeitpunkte als auch die Längsschnittanalyse von Erwerbsübergängen zulassen. Die Stichprobe umfasst für Westdeutschland knapp 13000 und für Ostdeutschland etwa 4500 Personen, die - jährlich wiederholt - unter anderem zu den Themen Beruf und Familie befragt werden. Als in Teilzeit tätig werden im Folgenden Beschäftigte mit einer wöchentlichen Arbeitszeit von höchstens 35 Stunden betrachtet, die sich selbst auf die Frage nach ihrer derzeitigen Erwerbstätigkeit als in Teilzeit oder geringfügig beschäftigt einstufen. Die auf dieser Grundlage berechneten Teilzeitquoten geben den jeweiligen Anteil der in Teilzeit Beschäftigten an allen Beschäftigten wieder. Die Bestimmungsgründe der Erwerbsübergänge von Frauen werden mittels logistischer Regressionsmodelle analysiert.

Der Forschungsstand zeigt: Das Hauptmotiv um in Teilzeit erwerbstätig zu sein ist, dass diese Erwerbsform nach wie vor als Möglichkeit zur Vereinbarkeit von Familie und Beruf angesehen wird. Eine ganze Reihe von Studien weist deshalb auf die Besonderheiten der Erwerbssituation von Frauen bzw. von Müttern hin. Insbesondere wird Teilzeitbeschäftigung von Müttern genutzt, um nach einer Familienphase wieder auf den Arbeitsmarkt zurückzukehren. Deutlich wird auch, dass sich bisherige Studien für Deutschland meist auf Westdeutschland beschränken. Darüber hinaus mangelt es an belastbaren Studien über teilzeitbeschäftigte Männer, obwohl anzunehmen ist, dass sich die Chancen bzw. Risiken, die Teilzeitbeschäftigung mit sich bringt, für verschiedene Gruppen von Beschäftigten und insbesondere für Männer und Frauen deutlich unterscheiden. Um zu beschreiben, wer Teilzeit arbeitet, werden Teilzeitquoten nach Geschlecht und Altersgruppen präsentiert und die Determinanten der Beschäftigungsübergänge von Frauen in Teilzeit und in Vollzeit vergleichend analysiert.

Obwohl auch die Teilzeitquoten der männlichen Beschäftigten gestiegen sind - fast jeder zehnte Arbeitnehmer und knapp die Hälfte der Arbeitnehmerinnen sind teilzeitbeschäftigt - stellen Frauen die überwiegende Mehrheit der Teilzeitbeschäftigten: vier von fünf Teilzeitbeschäftigten sind weiblich. Überdurchschnittlich häufig arbeiten Frauen über 35 Jahren in Teilzeit. Außerdem sind ältere Arbeitnehmer sowie Berufseinsteiger besonders von der Zunahme der Teilzeit betroffen. Als relevante Determinanten der Erwerbsübergänge in Teilzeitbeschäftigung zeigen sich in 
erster Linie familiale Bestimmungsgründe: Sowohl Mutterschaft als auch Ehe sprechen bei Frauen für die Aufnahme einer Teilzeitstelle. Allerdings nehmen ostdeutsche Frauen seltener eine Teilzeitstelle an als westdeutsche, und zwar auch dann, wenn sie Kinder haben. Darüber hinaus ist Teilzeitbeschäftigung für ostdeutsche Arbeitnehmerinnen häufiger ein Sprungbrett in eine Vollzeitanstellung. Offensichtlich kann Teilzeiterwerbstätigkeit der besseren Vereinbarkeit von Familie und Beruf dienen, allerdings wird dieses Arrangement eher von Frauen und Müttern in Westdeutschland gelebt.

Die Teilzeit ermöglicht also insbesondere jenen Arbeitsmarktgruppen die Erwerbstätigkeit, die aufgrund von Verpflichtungen in anderen Lebensbereichen nicht in Vollzeit partizipieren können oder wollen. Dabei eröffnet sie den Beschäftigten durchaus individuelle, über den Beruf vermittelte materielle und soziale Teilhabechancen. Ein eigenes Einkommen, individuelle Beiträge zu Arbeitslosen-, Kranken- und Rentenversicherung sowie soziale Integration über berufliche Kontakte sind gewährleistet. Folglich erscheint der Gedanke nahe liegend, das Potenzial der Teilzeitbeschäftigung könnte auch durch andere Arbeitsmarktgruppen zukünftig noch stärker genutzt werden, um die Belastung durch Erwerbsarbeit im Lebensverlauf umzuverteilen. Dadurch könnten Phasen der Ausbildung, der Familiengründung oder auch der Pflegearbeit erheblich entlastet oder Phasen der beruflichen Weiterentwicklung leichter eingeflochten werden. In solchen Situationen könnte Teilzeitarbeit eine Alternative auf Zeit zur Vollzeitbeschäftigung darstellen, und zwar für Frauen und Männer. Auch aufgrund der steigenden Lebenserwartung erscheint eine Umverteilung von bezahlter und unbezahlter Arbeit im Lebensverlauf sinnvoll. Beispielsweise kann Erwerbsarbeit mit Hilfe von Teilzeitbeschäftigung auf eine längere Lebensspanne umverteilt werden, die das bisherige Renteneintrittsalter von 65 bzw. zukünftig 67 Jahren möglicherweise weit überschreitet. Die entscheidende Frage bleibt aber, ob die durch Teilzeitbeschäftigung ermöglichten sozialen und materiellen Teilhabechancen ausreichend sind bzw. wie Teilzeitarbeitsverhältnisse ausgestaltet werden müssten, um diese zu gewährleisten ohne gleichzeitig bestehende soziale Ungleichheiten bspw. zwischen den Geschlechtern zu verschärfen.

\section{Executive Summary}

The standard continuous full-time employment relationship is associated with social and material opportunities as well as with social security that enable the employees to fully participate in societal life. Empirically, the labour market in Germany is predominated by such full-time standard employment relationships. Nevertheless, since the reunification at the latest we observe a continuous increase in part-time employment. Figures by the Statistisches Bundesamt show an increase in part-timers between 1991 and 2004 from 4.7 million to 7.2 million employees. That is about a quarter (23\%) of employees, while the part-time share in 1991 was only $14 \%$. On the basis of the German Socio-Economic Panel (GSOEP) from 1991 to 2006, we investigate how many women and men do work part-time, how participation in part-time employment varies by age groups and - estimating logistic regression models - which factors facilitate the transition to work either part-time or full-time amongst women.

The proportion of part-timers also increased amongst male employees - almost one in ten male employees is a part-timer and just under half of female employees work part-time - however, women are predominant amongst parttimers: four in five part-timers are female. Above-average shares of part-time employment show among women aged 36 and elder. Furthermore, elderly employees as well as labour market entrants have experienced a significant increase in the prevalence of part-time employment. For women, both motherhood and marriage increase the likelihood to work part-time. However, women from East Germany are less likely to work part-time than are those from West Germany, and that is also the case when they have children. In addition, part-time employment permits transitions into full-time positions more often amongst employees from East Germany. Apparently, part-time employment helps to combine family and working life continuously, but this arrangement is more frequently chosen by mothers in West Germany.

Part-time employment enables those labour market groups to participate that are due to various reasons unwilling or unable to work full-time. We find that part-time employment has the potential to bring even more individuals into paid work, also to manage a balance of family and employment obligations throughout the life course. However, part-time employment relationships also might contribute to increase social inequality especially between male and female employees.

\section{Literatur}

Allison, P.D.: Event History Analysis. Sage Publications, Newbury Park (1984)

Anger, S., Bowen, D.A., Engelmann, M., Frick, J.R., Goebel, J., Grabka, M.M., Groh-Samberg, O., Haas, H., Headey, B., Holst, E., Krause, P., Kroh, M., Kurka, C., Lohmann, H., Pischner, R., Rahmann, U., Schmitt, C., Schräpler, J.-P., Schupp, J., Sieber, I., Siedler, T., Spieß, C.K., Spieß, M., Tucci, I., Wagner, G.G.: 25 Wellen Sozio-oekonomisches Panel. Vierteljahresh. DIW Berlin 3, 9-14 (2008)

Blossfeld, H.-P., Hakim, C. (eds.) Between Equalization and Marginalization. Oxford University Press, Oxford (1997) 
Blossfeld, H.-P., Rohwer, G.: Part-Time Work in West Germany. In: Blossfeld, H.-P., Hakim, C. (eds.) Between Equalization and Marginalization, pp. 164-190. Oxford University Press, Oxford (1997)

Bothfeld, S.: Teilzeit für alle? Eine Untersuchung von Teilzeitpräferenzen in Deutschland und Großbritannien unter beschäftigungspolitischen Gesichtspunkten. Wissenschaftszentrum Berlin für Sozialforschung (FS I 97-205), Berlin (1997)

Bothfeld, S., O'Reilly, J.: Moving Up or Moving Out? Transitions through Part-Time Employment in Britain and Germany. In: O'Reilly, J., Cebrian, Inmaculada/Lallement, M. (eds.) Working Time Changes, pp. 132-171. Edward Elgar, Cheltenham (2000)

Bruegel, I., Hegewisch, A.: Flexibilization and Part-Time Work in Europe. In: Brown, P., Crompton, R. (eds.) Economic Restructuring and Social Exclusion, pp. 33-57. UCL Press, London (1994)

Büchel, F., Spieß, C.K.: Form der Kinderbetreuung und Arbeitsmarktverhalten von Müttern in West- und Ostdeutschland. Bundesministerium für Familie, Senioren, Frauen und Jugend, Berlin (2002)

Diaz-Bone, R.: Statistik für Soziologen. UVK, Konstanz (2006)

DeMaris, A.: Logit Modeling. Sage Publications, Newbury Park (1992)

Drobnic, S., Wittig I.: Vollzeit- und Teilzeiterwerbstätigkeit im Familienzyklus US-amerikanischer Frauen - Eine Längsschnittanalyse. Z. Soziol. 24, 374-389 (1995)

Engstler, H., Menning, S.: Die Familie im Spiegel der amtlichen Statistik. Bundesministerium für Familie, Senioren, Frauen und Jugend, Berlin (2003)

Giesecke, J.: Arbeitsmarktflexibilisierung und Soziale Ungleichheit. VS Verlag, Wiesbaden (2006)

Gustafsson, S.S., Wetzels, C.M.M.P., Vlasblom, J.D., Dex, S.: Women's Labor Force Transitions in Connection with Childbirth: A Panel Data Comparison between Germany, Sweden and Great Britain. J. Popul. Econ. 9, 223-246 (1996)

Hank, K., Kreyenfeld, M., Spieß, K.C.: Kinderbetreuung und Fertilität in Deutschland. Z. Soziol. 33, 228-244 (2004)

Holst, E., Maier, F.: Normalarbeitsverhältnis und Geschlechterordnung. Mitt. Arbeitsmarkt- und Berufsforsch. 31, 506-518 (1998)

Keller, B., Seifert, H.: Atypische Beschäftigungsverhältnisse: Flexibilität, soziale Sicherheit und Prekarität. WSI Mitt. 5, 235-240 (2006)

Künemund, H.: „Produktive“ Tätigkeiten. In: Kohli, M., Künemund, H. (Hrsg.) Die zweite Lebenshälfte - Gesellschaftliche Lage und Partizipation im Spiegel des Alters-Survey, S. 277-317. Leske + Budrich, Opladen (2000)

Kohler, H., Spitznagel, E.: Teilzeitarbeit in der Gesamtwirtschaft und aus der Sicht von Arbeitnehmern und Betrieben in der Bundesrepublik Deutschland. Mitt. Arbeitsmarkt- und Berufsforsch. 28, 339-364 (1995)

Lindecke, C., Voss-Dahm, D., Lehndorff, S.: Altersteilzeit (Arbeitspapier Nr. 142). Hans-Böckler-Stiftung, Düsseldorf (2007)

McRae, S.: Occupational Change over Childbirth. Sociology 25, 589-606 (1991)

O'Reilly, J., Fagan, C. (eds.) Part-time prospects: An international comparison of part-time work in Europe, North America and the Pacific Rim. Routledge, London (1998)
Quack, S.: Dynamik der Teilzeitarbeit: Implikationen für die soziale Sicherung von Frauen. edition sigma, Berlin (1993)

Schäfer, A., Vogel, C.: Teilzeitbeschäftigung als Arbeitsmarktchance. Wochenber. DIW Berlin 7, 131-138 (2005)

Scheuer, A., Dittmann, J.: Berufstätigkeit von Müttern bleibt kontrovers. Informationsdienst Soziale Indikatoren ISI 38, 1-5 (2007)

Schmal, A.: Teilzeitbeschäftigung. Motive, Präferenzen und Barrieren aus der Sicht von Arbeitnehmern. Campus, Frankfurt am Main (1997)

Schupp, J.: Teilzeitarbeit als Möglichkeit der beruflichen Re-Integration. In: Mayer, K. U., Allmendinger, J., Huinink, J. (Hrsg.) Vom Regen in die Traufe, S. 207-232. Campus, Frankfurt am Main (1991)

Statistisches Bundesamt: Leben und Arbeiten in Deutschland Mikrozensus 2004. Statistisches Bundesamt, Berlin (2005)

Stephan, G.: Zur Dynamik des Arbeitsangebotes von Frauen - Vollzeit-, Teilzeit- und Nichterwerbstätigkeit. Campus, Frankfurt am Main (1995)

Tam, M.: Part-time employment: A bridge or a trap? Ashgate Publishing Limited, Avebury (1997)

Tálos, E. (Hrsg.): Atypische Beschäftigung: Internationale Trends und sozialstaatliche Regelungen. Manz, Wien (1999)

Vaupel, J., Loichinger, E.: Redistributing Work in Aging Europe. Science 312, 1911-1913 (2006)

Vogel, C.: Flexible Beschäftigung und soziale Ungleichheit: Teilzeitbeschäftigung in Großbritannien und Deutschland im Vergleich. Humboldt-Universität zu Berlin, Berlin. http://edoc.hu-berlin.de/ docviews/abstract.php?lang=ger\&id=28019 (2007). Letzter $\mathrm{Zu}$ griff: 8 März 2009

Wagner, S.: Teilzeitarbeit. Ein Gesetz liegt im Trend (IAB-Kurzbericht 18/2004). Institut für Arbeitsmarkt- und Berufsforschung, Nürnberg (2004)

Waldfogel, J.: The Price of Motherhood: Family Status and Womens' Pay in a Young British Cohort. Oxf. Econ. Pap. 47, 584-610 (1995)

Ziefle, A.: Die individuellen Kosten des Erziehungsurlaubs. Kölner Z. Soziol. Sozialpsychol. 56, 213-231 (2007)

Claudia Vogel, Studium der Soziologie, Volkswirtschaftslehre und Neueren Geschichte an der Universität Potsdam und der University of Sheffield, Magistra Artium in Soziologie 2000. Bis 2003 wissenschaftliche Mitarbeiterin an der Humboldt-Universität zu Berlin, im Bereich Empirische Sozialforschung, Institut für Sozialwissenschaften. Von 2004 bis 2007 wissenschaftliche Mitarbeiterin der Freien Universität Berlin, in der Forschungsgruppe Altern und Lebenslauf (FALL) am Institut für Soziologie. 2006 Promotion zur Dr. phil. an der Humboldt-Universität zu Berlin. Seit Wintersemester 2007/2008 wissenschaftliche Mitarbeiterin der Hochschule Vechta, im Bereich Empirische Alternsforschung und Forschungsmethoden am Institut für Gerontologie sowie im Zentrum Altern und Gesellschaft.

Forschungsfelder: Generationenbeziehungen und intergenerationelle Transfers, über den Arbeitsmarkt sowie durch Vermögensübertragungen zwischen Generationen vermittelte soziale Ungleichheiten, international vergleichende Forschung mit Fokus auf westliche Wohlfahrtsregimes.

E-Mail: claudia.vogel@uni-vechta.de 